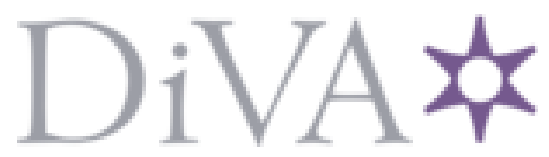

http://www.diva-portal.org

\title{
Postprint
}

This is the accepted version of a paper presented at IEEE Wireless Communications and Networking Conference (WCNC), Istanbul, Turkey.

Citation for the original published paper:

Shalmashi, S., Björnson, E., Slimane, B., Debbah, M. (2014)

Closed-Form Optimality Characterization of Network-Assisted Device-to-Device

Communications.

In: Proceedings of the IEEE Wireless Communications and Networking Conference (WCNC)

N.B. When citing this work, cite the original published paper.

Permanent link to this version:

http://urn.kb.se/resolve?urn=urn:nbn:se:kth:diva-139018 

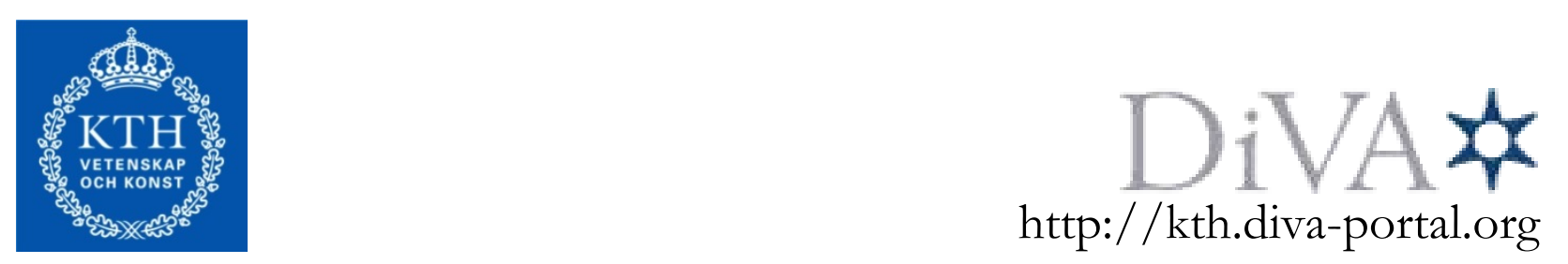

\section{Closed-Form Optimality Characterization of Network-Assisted Device-to-Device Communications}

IEEE Wireless Communication and Networking Conference (WCNC), Istanbul, Turkey, April 2014

(C) 2014 IEEE. Personal use of this material is permitted. Permission from IEEE must be obtained for all other uses, in any current or future media, including reprinting/republishing this material for advertising or promotional purposes, creating new collective works, for resale or redistribution to servers or lists, or reuse of any copyrighted component of this work in other works.

\section{SERVEH SHALMASHI, EMIL BJÖRNSON, SLIMANE BEN SLIMANE, AND MÉROUANE DEBBAH}

This is an author produced version of the paper.

Access to the published version may require subscription.

Published with permission from: IEEE 


\title{
Closed-Form Optimality Characterization of Network-Assisted Device-to-Device Communications
}

\author{
Serveh Shalmashi*, Emil Björnson ${ }^{\S \ddagger}$, Slimane Ben Slimane*, and Mérouane Debbah ${ }^{\ddagger}$ \\ *Department of Communication Systems, School of ICT, KTH Royal Institute of Technology, Stockholm, Sweden

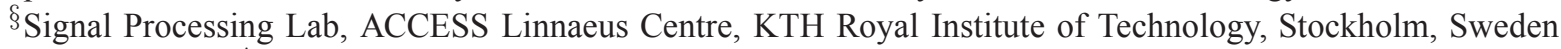 \\ $¥$ Alcatel-Lucent Chair on Flexible Radio, SUPELEC, Gif-sur-Yvette, France \\ E-mail: \{serveh,slimane\}@kth.se, \{emil.bjornson, merouane.debbah\}@supelec.fr
}

\begin{abstract}
This paper considers the mode selection problem for network-assisted device-to-device (D2D) communications with multiple antennas at the base station. We study transmission in both dedicated and shared frequency bands. Given the type of resources (i.e., dedicated or shared), the user equipment (UE) decides to transmit in the conventional cellular mode or directly to its corresponding receiver in the D2D mode. We formulate this problem under two different objectives. The first problem is to maximize the quality-of-service $(\mathrm{QoS})$ given a transmit power, and the second problem is to minimize the transmit power given a QoS requirement. We derive closed-form results for the optimal decision and show that the two problem formulations behave differently. Taking a geometrical approach, we study the area around the transmitter UE where the receiving UE should be to have D2D mode optimality, and how it is affected by the transmit power, QoS, and the number of base station antennas.
\end{abstract}

\section{INTRODUCTION}

Emerging multimedia services and applications introduce new traffic types and user behaviors [1]. To address the higher demands imposed on wireless networks, more spectrally efficient and energy efficient approaches should be developed. Device-to-device (D2D) communication underlaying cellular networks is proposed to improve cell spectral and energy efficiency of the network [2], [3]. In D2D transmission mode, user equipments (UEs) communicate directly to their intended receivers as opposed to the conventional cellular mode where they communicate through the base station (BS). D2D mode can bring proximity gains and reduce the transmission time.

Users in the D2D mode can transmit either in a separate frequency band or via spectrum sharing with cellular users. In the former case, D2D communications do not interfere with cellular users. This case is interesting due to its potential applications, such as public safety and multicasting for local multimedia services and robustness to infrastructure failure. On the other hand, spectrum sharing can be employed to efficiently utilize the resources which allows for better area spectral efficiency [4]. The gain from spectrum sharing can be assured if the interference is controlled by proper mode selection and resource management. However, depending on the network topology and channel conditions, it may not always be beneficial to choose the D2D mode for a UE.

E. Björnson is funded by the International Postdoc Grant 2012-228 from The Swedish Research Council. This research has been supported by the ERC Starting Grant 305123 MORE.
The studies [5] and [6] consider the mode selection problem with power control for one D2D user and one cellular UE (CUE) in a single antenna system. This problem corresponds to a choice between orthogonal resources, spectrum sharing, and conventional cellular transmission for the UE with D2D capability. In [5], the problem is formulated for two cases: greedy sum rate maximization where the CUE and D2D users are competing entities and sum rate maximization under a rate constraint for the CUE. Both problems are solved under power/energy constraints. In [6], a similar scenario is considered where the cell sum rate in single- and multi-cell scenarios is studied under a rate constraint that gives priority to the CUE. Moreover, [7] considers a scenario in which the position of the D2D transmitter and receiver are fixed, while the CUE's position can change. Two uplink transmission scenarios are investigated. In the former, the D2D user and the CUE share the spectrum if the D2D's signal-to-interferenceand-noise ratio (SINR) exceeds a pre-defined threshold. In the latter, the CUE can also use a relay to reduce its power while sharing the spectrum with the D2D user. In both cases, the D2D user's power is fixed and the interference from the D2D user to the BS is assumed negligible.

In this paper, we consider network-assisted D2D communications where the network helps the devices to perform peer discovery, radio resource management, and resolve security issues [1], [3]. Therefore, all UEs have the same priority in the network regardless of their mode of operation (i.e., cellular or D2D mode). This is different from prior works in which D2D users have lower priority and thus underlay cellular networks or, similarly, are considered secondary entities in a cognitive radio system. Network-assisted D2D communications can guarantee certain quality-of-service (QoS) levels for all UEs.

We define the mode selection problem as follows: given the type of resources, i.e., dedicated or shared, the UE decides which operation mode to select, i.e., the D2D or cellular mode. Furthermore, we take into account the effects of multiple antennas in the BS as it is an important feature of LTE and IMT-Advanced systems that enables simultaneous scheduling of spatially separated users [1], [8], [9]. The mode selection problem is formulated with two objectives: maximizing the QoS for a given transmit power, and minimizing the power for a given QoS. Our closed-form results show that the optimal 


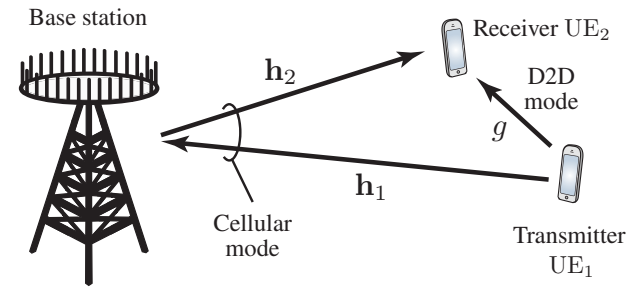

Fig. 1. Illustration of the system model where $\mathrm{UE}_{1}$ communicates with $\mathrm{UE}_{2}$, either via the BS (cellular mode) or by direct transmission (D2D mode).

decision based on these two formulations behaves differently. Using a geometrical approach, we study how the area of optimality for D2D transmission mode is affected by the transmit power, QoS, and the number of antennas at the BS.

\section{Problem Formulation}

We consider a cellular network where the single-antenna $\mathrm{UE}_{1}$ would like to communicate with the single-antenna $\mathrm{UE}_{2}$ located in the same cell. The BS of this cell is equipped with an array of $N$ antennas and takes care of the radio resource management. In the conventional cellular mode, $\mathrm{UE}_{1}$ first transmit its data to the BS using uplink resources and then the $\mathrm{BS}$ forwards the data to $\mathrm{UE}_{2}$ using downlink resources. However, when $\mathrm{UE}_{1}$ and $\mathrm{UE}_{2}$ are close to one another there is an alternative option: the $\mathrm{BS}$ allows $\mathrm{UE}_{1}$ to transmit directly to $\mathrm{UE}_{2}$ in a $D 2 D$ mode. This scenario is illustrated in Fig. 1. The main question that this paper tries to answer is: When is D2D mode preferable over cellular mode?

To make a fair comparison, the same amount of resources is allocated to each mode. We stress that $\mathrm{UE}_{1}$ transmits the whole time when in D2D mode as compared to half the time in cellular mode. This important difference is illustrated in Fig. 2. The D2D mode only uses uplink resources, while cellular mode uses both uplink and downlink resources. This has no importance in time-division duplex (TDD) systems, because the ratio of uplink to downlink resources is flexible. Our analysis is also applicable in frequency-division duplex (FDD) systems, except in extreme high-traffic situations.

\section{A. System Model}

Let $\mathbf{h}_{1} \in \mathbb{C}^{N \times 1}$ denote the channel between $\mathrm{UE}_{1}$ and the BS, while $\mathbf{h}_{2} \in \mathbb{C}^{N \times 1}$ is the channel between the BS and $\mathrm{UE}_{2}$. Also let $\mathbf{w}_{1} \in \mathbb{C}^{N \times 1}$ and $\mathbf{w}_{2} \in \mathbb{C}^{N \times 1}$ denote the unitnorm receiver and transmit beamforming vectors, respectively. The direct link between the UEs is $g \in \mathbb{C}$. See Fig. 1.

For fixed channel realizations, Gaussian codebooks, and perfect channel knowledge at the $\mathrm{BS}$ and $\mathrm{UE}_{2}$, the achievable spectral efficiencies (in bits per channel use) are

$$
\begin{gathered}
R_{\text {cell }}\left(p_{\mathrm{UE}}, p_{\mathrm{BS}}\right)=\frac{1}{2} \min \left(\log _{2}\left(1+\frac{p_{\mathrm{UE}} \kappa}{I_{\mathrm{BS}}+\sigma_{\mathrm{BS}}^{2}}\left|\mathbf{h}_{1}^{H} \mathbf{w}_{1}\right|^{2}\right),\right. \\
\left.\log _{2}\left(1+\frac{p_{\mathrm{BS}} \kappa}{I_{\mathrm{UE}}^{\mathrm{dl}}+\sigma_{\mathrm{UE}}^{2}}\left|\mathbf{h}_{2}^{H} \mathbf{w}_{2}\right|^{2}\right)\right) \\
R_{\mathrm{D} 2 \mathrm{D}}\left(p_{\mathrm{UE}}\right)=\log _{2}\left(1+\frac{p_{\mathrm{UE}}}{I_{\mathrm{UE}}+\sigma_{\mathrm{UE}}^{2}}|g|^{2}\right)
\end{gathered}
$$

for cellular mode and D2D mode, respectively. The transmit power of $\mathrm{UE}_{1}$ is $p_{\mathrm{UE}}$, while the transmit power of the $\mathrm{BS}$ is

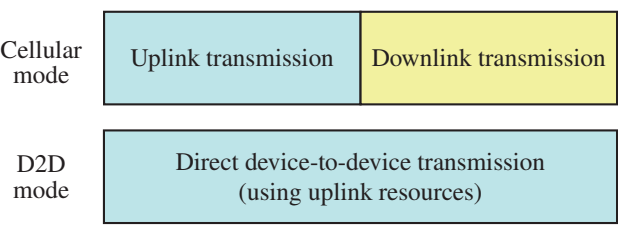

Fig. 2. By cutting out the middleman (the BS), D2D mode can effectively use twice the amount of resources for data transmission than cellular mode.

$p_{\mathrm{BS}}$. The parameter $\kappa$ decides whether the UE and BS can double the energy per channel use in cellular mode $(\kappa=2)$, since they only transmits half of the time, or if the energy is fixed $(\kappa=1)$. The additive circularly-symmetric complex Gaussian noise has variance $\sigma_{i}^{2}, i \in\{\mathrm{UE}, \mathrm{BS}\}$. The term $I_{\mathrm{UE}}^{\mathrm{dl}}$ is the estimated interference power at $\mathrm{UE}_{2}$ in the downlink. The terms $I_{i}, i \in\{\mathrm{UE}, \mathrm{BS}\}$ are the estimated interference powers at $\mathrm{UE}_{2}$ and the $\mathrm{BS}$ in the uplink, which are created by other cellular or D2D users' transmissions when the allocated resource is shared. Consequently, there is no interference when dedicated resources are reserved for D2D communications.

Assumption 1. The BS has much larger power resources and interference rejection capability than $\mathrm{UE}_{1}$, thus it can make $\log _{2}\left(1+\frac{p_{\mathrm{BS}} \kappa}{I_{\mathrm{UF}}^{\mathrm{II}}+\sigma_{\mathrm{UF}}^{2}}\left|\mathbf{h}_{2}^{H} \mathbf{w}_{2}\right|^{2}\right) \geq \log _{2}\left(1+\frac{p_{\mathrm{UE}} \kappa}{I_{\mathrm{BS}}+\sigma_{\mathrm{SS}}^{2}}\left|\mathbf{h}_{1}^{H} \mathbf{w}_{1}\right|^{2}\right)$ for any $\mathbf{h}_{1}, \mathbf{h}_{2}$. Consequently, we assume that (1) can be replaced by

$$
R_{\mathrm{cell}}\left(p_{\mathrm{UE}}\right)=\frac{1}{2} \log _{2}\left(1+\frac{p_{\mathrm{UE}} \kappa}{I_{\mathrm{BS}}+\sigma_{\mathrm{BS}}^{2}}\left|\mathbf{h}_{1}^{H} \mathbf{w}_{1}\right|^{2}\right) .
$$

In other words, we assume that the uplink transmission is the limiting factor in the cellular mode.

There are two main approaches to optimize the resource allocation of the transmission. The first one is to maximize the spectral efficiency, or QoS, under a given transmit power $p_{\mathrm{UE}}=p_{\mathrm{UE}}^{*}$. This is stated mathematically as

$$
\begin{array}{ll}
\underset{R}{\operatorname{maximize}} & R \\
\text { subject to } & \max \left(R_{\text {cell }}\left(p_{\mathrm{UE}}^{*}\right), R_{\mathrm{D} 2 \mathrm{D}}\left(p_{\mathrm{UE}}^{*}\right)\right) \geq R .
\end{array}
$$

Alternatively, one can minimize the transmit power required to maintain a given QoS level $R^{*}$. This is stated as

$$
\begin{array}{ll}
\underset{p_{\mathrm{UE}}}{\operatorname{minimize}} & p_{\mathrm{UE}} \\
\text { subject to } & \max \left(R_{\text {cell }}\left(p_{\mathrm{UE}}\right), R_{\mathrm{D} 2 \mathrm{D}}\left(p_{\mathrm{UE}}\right)\right) \geq R^{*} .
\end{array}
$$

As shown in [8], the optimization problems (P1) and (P2) are tightly connected [8]: let the optimal solution to (P1) with transmit power $p_{\mathrm{UE}}^{*}$ be denoted $\tilde{R}^{*}$, then the optimal solution to (P2) with the QoS level $\tilde{R}^{*}$ is exactly $p_{\mathrm{UE}}^{*}$. Nevertheless, we show that these optimization problems behave differently in terms of when D2D mode is preferable over cellular mode, and vice versa. The analysis is provided in the next two sections.

\section{Maximize Spectral Efficiency: D2D Optimality}

The optimization problem (P1) can be solved directly by computing $\max \left(R_{\text {cell }}\left(p_{\mathrm{UE}}^{*}\right), R_{\mathrm{D} 2 \mathrm{D}}\left(p_{\mathrm{UE}}^{*}\right)\right)$ and assigning this value to $R$. What we would like to derive is a condition on when $R_{\mathrm{D} 2 \mathrm{D}}\left(p_{\mathrm{UE}}^{*}\right) \geq R_{\text {cell }}\left(p_{\mathrm{UE}}^{*}\right)$; that is, when $\mathrm{D} 2 \mathrm{D}$ mode is preferably from a QoS viewpoint. First, the optimization problem (P1) is solved when a dedicated resource is available for the UE, then we extend the results for spectrum sharing. 


\section{A. D2D Optimality with a Dedicated Resource}

In the dedicated resource scenario, we assume the use of optimal maximum ratio combining for reception at the $\mathrm{BS}$ in cellular mode: $\mathbf{w}_{1}^{\mathrm{MRC}}=\frac{\mathbf{h}_{1}}{\left\|\mathbf{h}_{1}\right\|}$. Therefore $\left|\mathbf{h}_{1}^{H} \mathbf{w}_{1}\right|^{2}=\left\|\mathbf{h}_{1}\right\|^{2}$. Based on Assumption 1, D2D optimality is equivalent to

$$
\begin{aligned}
\log _{2}\left(1+\frac{p_{\mathrm{UE}}^{*}}{\sigma_{\mathrm{UE}}^{2}}|g|^{2}\right) & \geq \frac{1}{2} \log _{2}\left(1+\frac{p_{\mathrm{UE}}^{*} \kappa}{\sigma_{\mathrm{BS}}^{2}}\left\|\mathbf{h}_{1}\right\|^{2}\right) \Leftrightarrow \\
\left\|\mathbf{h}_{1}\right\|^{2} & \leq \frac{2 \sigma_{\mathrm{BS}}^{2}}{\sigma_{\mathrm{UE}}^{2} \kappa}|g|^{2}+\frac{p_{\mathrm{UE}}^{*} \sigma_{\mathrm{BS}}^{2}}{\left(\sigma_{\mathrm{UE}}^{2}\right)^{2} \kappa}|g|^{4} .
\end{aligned}
$$

Since $\kappa \in\{1,2\}$, the condition (4) is trivially satisfied whenever the direct channel is stronger than the channel to the BS (i.e., when $|g|^{2} \geq\left\|\mathbf{h}_{1}\right\|^{2}$ ). However, the second term in (4) implies that D2D mode can be optimal also when the direct channel is weaker than than the channel to the BS, given that $p_{\mathrm{UE}}^{*}$ is large enough. To understand when this occurs, we solve (4) as a quadratic equation in $|g|^{2}$, which gives

$$
|g|^{2} \geq \sqrt{\frac{\left\|\mathbf{h}_{1}\right\|^{2}\left(\sigma_{\mathrm{UE}}^{2}\right)^{2} \kappa}{\sigma_{\mathrm{BS}}^{2} p_{\mathrm{UE}}^{*}}+\left(\frac{\sigma_{\mathrm{UE}}^{2}}{p_{\mathrm{UE}}^{*}}\right)^{2}}-\frac{\sigma_{\mathrm{UE}}^{2}}{p_{\mathrm{UE}}^{*}} .
$$

The inequalities (4) and (5) provide two equivalent closedform necessary and sufficient conditions for optimality of D2D mode in (P1). The following theorem provides a sufficient condition that is more amenable to explicit analysis.

Theorem 1 . The solution to (P1) is achieved by $\mathrm{D} 2 \mathrm{D}$ mode if

$$
|g|^{2} \geq \sqrt{\frac{\left\|\mathbf{h}_{1}\right\|^{2}\left(\sigma_{\mathrm{UE}}^{2}\right)^{2} \kappa}{\sigma_{\mathrm{BS}}^{2} p_{\mathrm{UE}}^{*}}} .
$$

Proof: The right-hand side of (5) satisfies

$$
\sqrt{\frac{\left\|\mathbf{h}_{1}\right\|^{2}\left(\sigma_{\mathrm{UE}}^{2}\right)^{2} \kappa}{\sigma_{\mathrm{BS}}^{2} p_{\mathrm{UE}}^{*}}+\left(\frac{\sigma_{\mathrm{UE}}^{2}}{p_{\mathrm{UE}}^{*}}\right)^{2}}-\frac{\sigma_{\mathrm{UE}}^{2}}{p_{\mathrm{UE}}^{*}} \leq \sqrt{\frac{\left\|\mathbf{h}_{1}\right\|^{2}\left(\sigma_{\mathrm{UE}}^{2}\right)^{2} \kappa}{\sigma_{\mathrm{BS}}^{2} p_{\mathrm{UE}}^{*}}},
$$

thus the condition (6) always implies that (5) is satisfied.

Several important conclusions are drawn from Theorem 1. Firstly, increasing the UE power $p_{\mathrm{UE}}^{*}$ makes D2D mode attractive also for weaker direct channels. This is explained by the fact that we then operate in the concave regime of the $\log _{2}(1+\mathrm{SNR})$-formula where it is costly to compensate for the $\frac{1}{2}$ prelog-factor of cellular mode by increasing the power. Secondly, the channel gain $\left\|\mathbf{h}_{1}\right\|^{2}$ should be proportional to the squared channel gain $\left(|g|^{2}\right)^{2}$ to choose cellular mode.

Remark 1 (Implementation Guidelines). The condition in Theorem 1 provides a simple mean to implement networkassisted D2D communication. Whenever a BS observes that $\mathrm{UE}_{1}$ and $\mathrm{UE}_{2}$ are in the same cell, $\mathrm{UE}_{1}$ is informed about it. $\mathrm{UE}_{1}$ will now listen to the uplink pilot signals sent by $\mathrm{UE}_{2}$ and thereby obtain $|g|^{2}$. The channel gain $\left\|\mathbf{h}_{1}\right\|^{2}$ is obtained in the conventional way used for cellular mode. Thus, $\mathrm{UE}_{1}$ can evaluate either (4), (5), or (6) and decide which mode is preferable for the moment. The fact that (6) only provides a sufficient condition can actually be a feature, because it removes special cases when D2D mode is only slightly better than cellular mode but not enough to motivate the extra overhead signaling.

\section{B. D2D Optimality with a Shared Resource}

So far, we have considered a scenario in which a dedicated channel is allocated to the UE. However, if the user shares the spectrum with other UEs which are spatially separated, there are manifold gains in cell spectral efficiency. To address spectrum sharing, it is assumed that each receiver can measure the interference power and treat the interference as noise.

Let $I_{\mathrm{ul}}=I_{\mathrm{BS}}+\sigma_{\mathrm{BS}}^{2}$ and $I_{\mathrm{D} 2 \mathrm{D}}=I_{\mathrm{UE}}+\sigma_{\mathrm{UE}}^{2}$. In order to solve the optimization problem (P1), we follow the same approach as in Section III-A. The equivalent of D2D condition (4) becomes

$$
\left|\mathbf{h}_{1}^{H} \mathbf{w}_{1}\right|^{2} \leq \frac{2}{\kappa} \frac{I_{\mathrm{ul}}}{I_{\mathrm{D} 2 \mathrm{D}}}|g|^{2}+\frac{p_{\mathrm{UE}}^{*}}{\kappa} \frac{I_{\mathrm{ul}}}{I_{\mathrm{D} 2 \mathrm{D}}^{2}}|g|^{4} .
$$

Besides the conclusions in Section III-A, (8) shows that D2D mode is optimal when the interference received at $\mathrm{UE}_{2}$ is much smaller than the one received at the base station, i.e., $I_{\mathrm{D} 2 \mathrm{D}} \ll I_{\mathrm{ul}}$. By solving (8) as a quadratic equation, we have

$$
|g|^{2} \geq \sqrt{\frac{\left|\mathbf{h}_{1}^{H} \mathbf{w}_{1}\right|^{2} I_{\mathrm{D} 2 \mathrm{D}}^{2} \kappa}{p_{\mathrm{UE}}^{*} I_{\mathrm{ul}}}+\left(\frac{I_{\mathrm{D} 2 \mathrm{D}}}{p_{\mathrm{UE}}^{*}}\right)^{2}}-\frac{I_{\mathrm{D} 2 \mathrm{D}}}{p_{\mathrm{UE}}^{*}} .
$$

Similar to Theorem 1, a simple sufficient condition for D2D mode optimality is

$$
|g|^{2} \geq \sqrt{\frac{\left|\mathbf{h}_{1}^{H} \mathbf{w}_{1}\right|^{2} I_{\mathrm{D} 2 \mathrm{D}}^{2} \kappa}{p_{\mathrm{UE}}^{*} I_{\mathrm{ul}}}}
$$

An important conclusion from (10) is that $\mathrm{UE}_{2}$ is more sensitive to interference when operating in the D2D mode. Therefore, the allocation of dedicated resources for D2D mode is of interest in rescue operations or local entertainment services. Such services might operate with multi-casting. It is straightforward to extend our optimality conditions to the multi-cast scenario by replacing $|g|^{2}$ by $\min _{k \in \mathcal{K}}\left|g_{k}\right|^{2}$, where $\mathcal{K}$ is the set of users that should receive the signal. However, we do not discuss this case further due to space limitations.

\section{Minimize Transmit Power: D2D Optimality}

In the following, we derive D2D optimality conditions for the optimization problem (P2). We observe that the spectral efficiencies $R_{\text {cell }}\left(p_{\mathrm{UE}}\right)$ and $R_{\mathrm{D} 2 \mathrm{D}}\left(p_{\mathrm{UE}}\right)$ increase monotonically with $p_{\mathrm{UE}}$, thus (P2) is solved when the QoS constraint holds with equality. The smallest transmit power that achieves $R^{*}$ can be computed explicitly for each mode.

\section{A. D2D Optimality with a Dedicated Resource}

Based on Assumption 1, we have in cellular mode that

$$
\frac{1}{2} \log _{2}\left(1+\frac{p_{\mathrm{UE}} \kappa}{\sigma_{\mathrm{BS}}^{2}}\left\|\mathbf{h}_{1}\right\|^{2}\right)=R^{*} \Rightarrow p_{\mathrm{UE}}=\left(2^{2 R^{*}}-1\right) \frac{\sigma_{\mathrm{BS}}^{2}}{\left\|\mathbf{h}_{1}\right\|^{2} \kappa} .
$$

The corresponding expression for D2D mode is

$$
\log _{2}\left(1+\frac{p_{\mathrm{UE}}}{\sigma_{\mathrm{UE}}^{2}}|g|^{2}\right)=R^{*} \Rightarrow p_{\mathrm{UE}}=\left(2^{R^{*}}-1\right) \frac{\sigma_{\mathrm{UE}}^{2}}{|g|^{2}}
$$

The solution to (P2) is obtained by taking the smallest value of (11) and (12). In other words, D2D mode is optimal whenever 
the required UE power for D2D mode in (12) is smaller than the power of cellular mode in (11). This is equivalent to

$$
\left(2^{R^{*}}-1\right) \frac{\sigma_{\mathrm{UE}}^{2}}{|g|^{2}} \leq\left(2^{2 R^{*}}-1\right) \frac{\sigma_{\mathrm{BS}}^{2}}{\left\|\mathbf{h}_{1}\right\|^{2} \kappa}
$$

and gives the following the conditions for D2D optimality.

Theorem 2. For a given $R^{*}>0$, the solution to (P2) is achieved by D2D mode if and only if

$$
|g|^{2} \geq \frac{1}{2^{R^{*}}+1} \frac{\sigma_{\mathrm{UE}}^{2}}{\sigma_{\mathrm{BS}}^{2}}\left\|\mathbf{h}_{1}\right\|^{2} \kappa .
$$

Hence, D2D mode is optimal for the QoS level $R^{*}$ if and only if

$$
R^{*} \geq \log _{2}\left(\frac{\sigma_{\mathrm{UE}}^{2}}{\sigma_{\mathrm{BS}}^{2}} \frac{\left\|\mathbf{h}_{1}\right\|^{2} \kappa}{|g|^{2}}-1\right) .
$$

Proof: The condition in (14) is achieved directly from (13) by noting that $\frac{2^{2 R^{*}}-1}{2^{R^{*}}-1}=\frac{\left(2^{R^{*}}-1\right)\left(2^{R^{*}}+1\right)}{2^{R^{*}}-1}=2^{R^{*}}+1$. The QoS condition in (15) is achieved by solving (14) for $R^{*}$.

This theorem proves that $\mathrm{D} 2 \mathrm{D}$ mode is optimal whenever the rate is above the threshold in (15). Somewhat surprisingly, this means that D2D mode is always optimal if we let $R^{*} \rightarrow$ $\infty$, irrespective of how weak the direct channel is but possibly at the expense of spending a lot of power. The threshold is negative for $|g|^{2}>\kappa\left\|\mathbf{h}_{1}\right\|^{2}$ thus D2D mode is always optimal when the direct channel is stronger than the channel to the BS - this is consistent with our observations in Section III-A.

\section{B. D2D Optimality with a Shared Resource}

In the case of spectrum sharing, the solution to (P2) for a given $R^{*}$ is achieved by D2D mode if and only if

$$
|g|^{2} \geq \frac{1}{2^{R^{*}}+1} \frac{I_{\mathrm{D} 2 \mathrm{D}}}{I_{\mathrm{ul}}}\left|\mathbf{h}_{1}^{H} \mathbf{w}_{1}\right|^{2} \kappa .
$$

Equivalently, D2D mode is optimal for the QoS levels

$$
R^{*} \geq \log _{2}\left(\frac{I_{\mathrm{D} 2 \mathrm{D}}}{I_{\mathrm{ul}}} \frac{\left|\mathbf{h}_{1}^{H} \mathbf{w}_{1}\right|^{2} \kappa}{|g|^{2}}-1\right) .
$$

\section{Geometrical Insights}

To gain some geometrical insights on the optimality of D2D mode, we now consider a simple path-loss model

$$
\begin{aligned}
|g|^{2} & =c_{g} d_{g}^{-b_{g}} \\
\left\|\mathbf{h}_{1}\right\|^{2} & =N c_{h} d_{h}^{-b_{h}}
\end{aligned}
$$

where $d_{g}, d_{h}$ are the distances between $\mathrm{UE}_{1}$ and $\mathrm{UE}_{2}$ and between $\mathrm{UE}_{1}$ and the $\mathrm{BS}$, respectively. Furthermore, $c_{g}, c_{h}, b_{g}, b_{h}>0$ are some arbitrary path-loss parameters.

\section{A. Maximize Spectral Efficiency}

Plugging this path-loss model into the optimality condition for D2D mode (6) for (P1) with dedicated resources yields

$$
\frac{d_{g}^{-b_{g}}}{d_{h}^{-\frac{b_{h}}{2}}} \geq \sqrt{\frac{N}{\sigma_{\mathrm{BS}}^{2} p_{\mathrm{UE}}^{*}}} \frac{\sigma_{\mathrm{UE}}^{2} \sqrt{\kappa c_{h}}}{c_{g}}
$$

where it is clear that increasing $p_{\mathrm{UE}}^{*}$ will make D2D mode more probable. This effect is counteracted by increasing the number of antennas $N$, which is explained by the array gain that is achieved by coherent beamforming at the BS.

For a fixed distance $d_{h}$ between $\mathrm{UE}_{1}$ and the $\mathrm{BS}$, we can compute the circular area $A$ around $\mathrm{UE}_{1}$ where $\mathrm{UE}_{2}$ (or all receivers in multi-casting) should be to enable D2D mode. From (20), we have the optimality condition

$$
A=\pi d_{g}^{2} \leq \pi d_{h}^{\frac{b_{h}}{b_{g}}}\left(\frac{p_{\mathrm{UE}}^{*}}{N} \frac{\sigma_{\mathrm{BS}}^{2} c_{g}^{2}}{\left(\sigma_{\mathrm{UE}}^{2}\right)^{2} \kappa c_{h}}\right)^{\frac{1}{b_{g}}} .
$$

This area increases with the distance from the BS (e.g., linearly for $b_{h}=b_{g}$ ), thus D2D mode is more probable in large macro cells and/or when $\mathrm{UE}_{1}$ is located at the cell edge. Moreover, the area grows with the transmit power as $\left(p_{\mathrm{UE}}^{*}\right)^{1 / b_{g}}$ and decreases as $1 / N^{1 / b_{g}}$ with the number of antennas.

In the shared spectrum case, we assume zero-forcing (ZF) beamforming at the BS to cancel the interference: $I_{\mathrm{BS}}=0$. This comes at the expense of the average SNR loss $\left|\mathbf{h}_{1}^{H} \mathbf{w}_{1}\right|^{2}=$ $\frac{N-M}{N}\left\|\mathbf{h}_{1}\right\|^{2}=(N-M) c_{h} d_{h}^{-b_{h}}$, where $M(M<N)$ is the number of interferers. The interference experienced by $\mathrm{UE}_{2}$ and its distance from $\mathrm{UE}_{1}$ depend on its coordinates $\left(x_{r}, y_{r}\right)$. Then, from (10) we have the D2D optimality condition

$$
I_{\mathrm{D} 2 \mathrm{D}}\left(x_{r}, y_{r}\right) \leq \sqrt{\frac{p_{\mathrm{UE}}^{*} \sigma_{\mathrm{BS}}^{2} c_{g}^{2}}{(N-M) \kappa c_{h}} \frac{d_{h}^{b_{h}}}{d_{g}^{2 b_{g}}\left(x_{r}, y_{r}\right)}} .
$$

\section{B. Minimize Transmit Power}

To gain some geometrical insight for (P2), we substitute (18) and (19) into (15). Then, we have the optimality condition

$$
\frac{d_{g}^{b_{g}}}{d_{h}^{b_{h}}} \leq \frac{\sigma_{\mathrm{BS}}^{2}}{\sigma_{\mathrm{UE}}^{2}} \frac{c_{g}\left(2^{R^{*}}+1\right)}{N c_{h} \kappa} .
$$

For a fixed $d_{h}$, The circular area around $\mathrm{UE}_{1}$ where $\mathrm{UE}_{2}$ (and other potential multi-cast receivers) should be is

$$
A=\pi d_{g}^{2} \leq \pi d_{h}^{\frac{2 b_{h}}{b_{g}}}\left(\frac{\sigma_{\mathrm{BS}}^{2}}{\sigma_{\mathrm{UE}}^{2}} \frac{c_{g}}{N} \frac{\left(2^{R^{*}}+1\right)}{\kappa c_{h}}\right)^{\frac{2}{b_{g}}} .
$$

The area of D2D mode depends on three factors: $d_{h}^{2 b_{h} / b_{g}}$, $\left(2^{R^{*}}+1\right)^{2 / b_{g}}$, and $N^{2 / b_{g}}$. The area increases by the first two factors but is inversely proportional to the last factor. In the shared spectrum case with ZF reception at the BS, the D2D mode is optimal if the interference in the $\mathrm{UE}_{2}$ is bounded as

$$
I_{\mathrm{D} 2 \mathrm{D}}\left(x_{r}, y_{r}\right) \leq \frac{\left(2^{R^{*}}+1\right) \sigma_{\mathrm{BS}}^{2} c_{g}}{(N-M) \kappa c_{h}} \frac{d_{h}^{b_{h}}}{d_{g}^{b_{g}}\left(x_{r}, y_{r}\right)} \text {. }
$$

\section{Numerical StUdy}

In this section, we evaluate the optimal mode selection for different system parameters by using Monte-Carlo simulations. A single circular cell with radius $R$ is considered where the BS is located in the middle. The distance of the D2D transmitter $\mathrm{UE}_{1}$ from the $\mathrm{BS}$ is fixed to $R / 2$. Different locations for the D2D receiver are considered with a minimum distance $d_{\min }$ from $\mathrm{UE}_{1}$ and from the $\mathrm{BS}$. The simulation parameters are given in Table I. The channel model accounts for the effects of path-loss and multi-path fading. The path-loss parameters 
TABLE I

SIMULATION PARAMETERS.

\begin{tabular}{lcc}
\hline Description & Parameter & Value \\
\hline UE transmit power & $p_{\mathrm{UE}}^{*}$ & $15 \mathrm{dBm}$ \\
QoS & $R^{*}$ & $\{2,4,6,8\} \mathrm{bpcu}$ \\
Nr. of antennas at BS & $N$ & $\{2,8,100\}$ \\
Nr. of interferers & $M$ & 7 \\
Cell radius & $R$ & $500 \mathrm{~m}$ \\
Noise power & $N_{0}$ & $-107 \mathrm{dBm}$ \\
Noise figure at UEs & $F$ & $5 \mathrm{~dB}$ \\
Carrier frequency & $f_{c}$ & $2 \mathrm{GHz}$ \\
System bandwidth & $B$ & $5 \mathrm{MHz}$ \\
Min. D2D receiver distance & $d_{\min }$ & $10 \mathrm{~m}$ \\
Path-loss exp. UE $\leftrightarrow \mathrm{UE}$ & $b_{g}$ & 4 \\
Path-loss exp. BS $\leftrightarrow \mathrm{UE}$ & $b_{h}$ & 3.67 \\
Path-loss coeff. UE $\leftrightarrow \mathrm{UE}$ & $c_{g}$ & $28.03 \mathrm{~dB}$ \\
Path-loss coeff. BS $\leftrightarrow \mathrm{UE}$ & $c_{h}$ & $30.55 \mathrm{~dB}$ \\
Monte-Carlo realizations & $\mathrm{MC}$ & 10000 \\
\hline
\end{tabular}

are based on the non-line-of-sight (NLoS) scenario in [10]. We assume Rayleigh block-fading channels where the channels are constant during one time slot, but vary between different time slots. Each receiver knows its channel. The average UE power is the same in both modes (i.e., $\kappa=2$ ) in our simulations.

\section{A. Dedicated Resource Scenario}

The scenario when dedicated resources are allocated to $\mathrm{UE}_{1}$ is considered in Fig. 3. The top plot shows results for (P1) and the bottom plot considers (P2). The dashed circles in Fig. 3 depict the D2D optimality areas derived in (21) and (24), respectively. While these expressions only consider path-losses (i.e., the average channel gains), the corresponding probabilistic areas obtained under Rayleigh fading are also illustrated in Fig. 3. We observe that the optimal area for D2D mode is much larger when the objective is maximizing the QoS as in (P1), as compared to minimizing the transmit power as in (P2). This is explained by the fact that the solution to (P1) operates at full power and thus D2D transmission has the huge benefit of using all its resources for UE transmission, instead of half of them as in cellular mode.

To dig deeper into the results, Fig. 4 shows the radius of the D2D optimality area for (P1) versus the number of antennas and different transmit powers. As proved in the analytical part, the area of optimality increases with the power. However, the area is reduced as the number of antennas is increased. In Fig. 5, for (P2), the D2D optimality region also becomes small if the QoS constraint is small and when the number of antennas is large. This confirms our analytic results as well.

\section{B. Spectrum Sharing Scenario}

In the spectrum sharing scenario, in addition to $\mathrm{UE}_{1}$, there exist $M$ interfering UEs equally distanced from the BS on a circle of radius $R / 2$. For the $\mathrm{D} 2 \mathrm{D}$ receiver, we considered a grid of possible positions separated by $5 \mathrm{~m}$ in the cell area.

Fig. 6 and Fig. 7 show the optimal mode of each receiver position based on the bounds derived in (22) and (25) using only path-loss information. In the presence of interference, the D2D optimality region in (P1) is larger than the corresponding region in (P2). In order to combat the interference, the $\mathrm{D} 2 \mathrm{D}$ transmitter needs to increase the power. Therefore, in (P2),
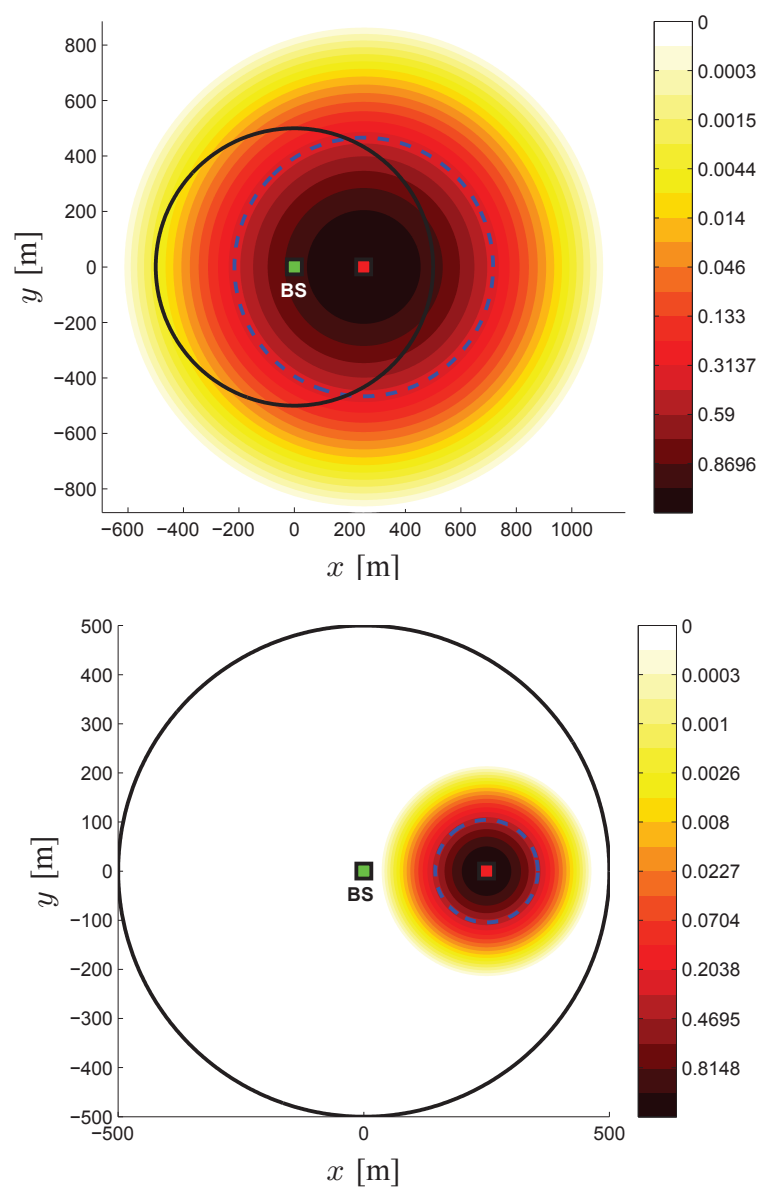

Fig. 3. Probability of D2D mode optimality for (P1) (top) and (P2) (bottom) with $N=8, p_{\mathrm{UE}}^{*}=15 \mathrm{dBm}, R^{*}=4$, and $d_{h}=250 \mathrm{~m}$. The color shows the probability for D2D mode optimality when the receiving UE is at different locations. The black circle is the cell boundary and the dashed blue one is the boundary of D2D optimality area based on only path-loss information.

it may be better to communicate through the BS rather than direct transmission. Note that the interference at the BS and D2D receiver have the same importance in (P2).

Figs. 8-9 consider fading channels. The probability of D2D mode optimality is higher when the receivers are farther away from the sources of interference and closer to their transmitter as it is shown in Fig. 9. The areas with distance less than $d_{\text {min }}$ to the D2D transmitter and the BS are excluded.

\section{CONCLUSiOnS}

We investigated the problem of mode selection for networkassisted D2D communications in single-cell scenarios with multiple antennas at the BS. We formulated the problem with two objectives: (P1) maximize QoS or (P2) minimize power. We derived closed-form conditions for the optimality of D2D mode in both cases. The analytic results are evaluated and illustrated by means of Monte-Carlo simulations. Our results show that the two problems have distinct differences in the resulting area of optimality for the D2D mode. Increasing the transmit power in (P1) or the QoS in (P2) increases the area of D2D optimality, because the D2D mode then benefits greatly from its better pre-log factor. However, increasing the number of antennas has the opposite effect. The results are easily extended to multi-casting scenarios. 


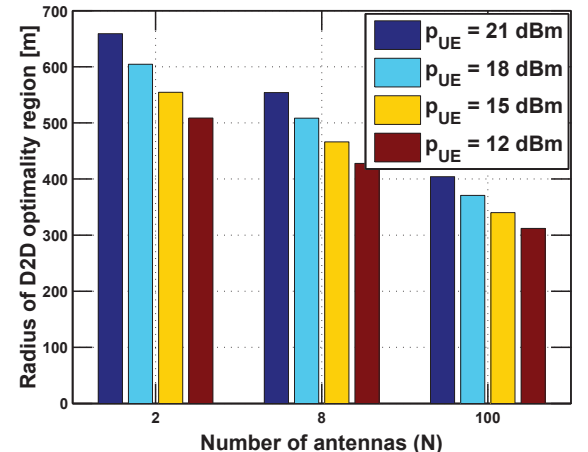

Fig. 4. Radius of the circular D2D optimality region vs. number of antennas for (P1) with dedicated resources.

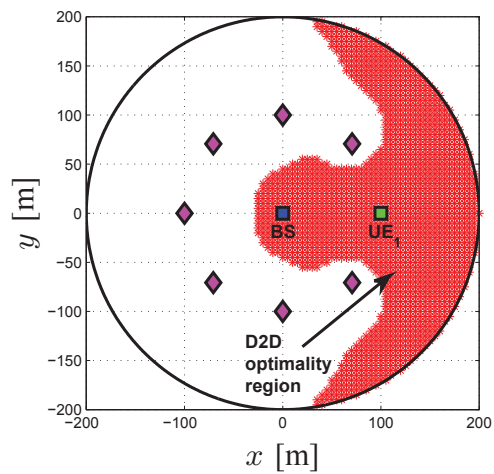

Fig. 6. D2D optimality area in the shared spectrum case with only path-loss information for ( $\mathrm{P} 1)$ with $N=8$ and $M=7$.

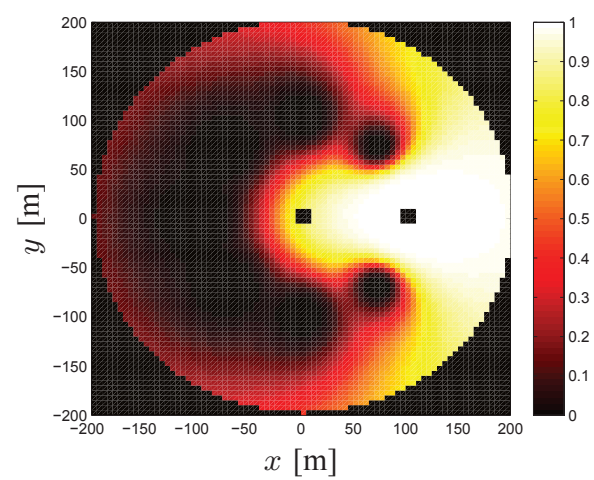

Fig. 8. Probability of D2D mode optimality in the shared spectrum case with fading for (P1) with $N=8$ and $M=7$.

\section{REFERENCES}

[1] R. Baldemair, E. Dahlman, G. Fodor, G. Mildh, S. Parkvall, Y. Selen, $\mathrm{H}$. Tullberg, and K. Balachandran, "Evolving wireless communications: Addressing the challenges and expectations of the future," IEEE Veh. Technol. Mag., vol. 8, no. 1, pp. 24-30, Mar. 2013.

[2] K. Doppler, M. Rinne, C. Wijting, C. Ribeiro, and K. Hugl, "Deviceto-device communication as an underlay to LTE-advanced networks," IEEE Commun. Mag., vol. 47, no. 12, pp. 42-49, Dec. 2009.

[3] G. Fodor, E. Dahlman, G. Mildh, S. Parkvall, N. Reider, G. Miklós, and Z. Turányi, "Design aspects of network assisted device-to-device communications," IEEE Commun. Mag., vol. 50, no. 3, pp. 170-177, Mar. 2012.

[4] G. Fodor and N. Reider, "A distributed power control scheme for cellular network assisted d2d communications," in Proc. IEEE Global Telecommun. Conf. (GLOBECOM), Houston, TX, Dec. 2011.

[5] C. Yu, K. Doppler, C. Ribeiro, and O. Tirkkonen, "Resource sharing optimization for device-to-device communication underlaying cellular networks," IEEE Trans. Wireless Commun., vol. 10, no. 8, pp. 27522763, Aug. 2011

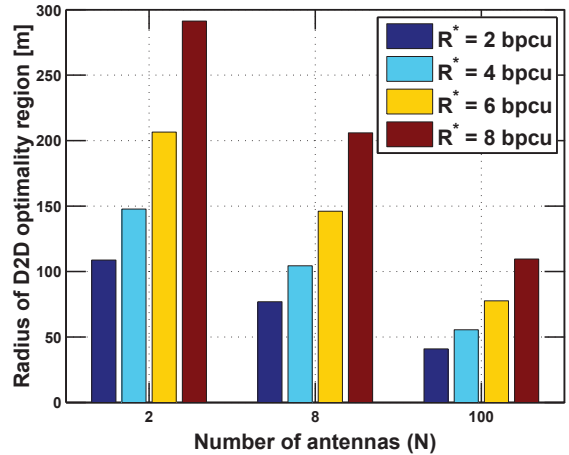

Fig. 5. Radius of the circular D2D optimality region vs. number of antennas for (P2) with dedicated resources.

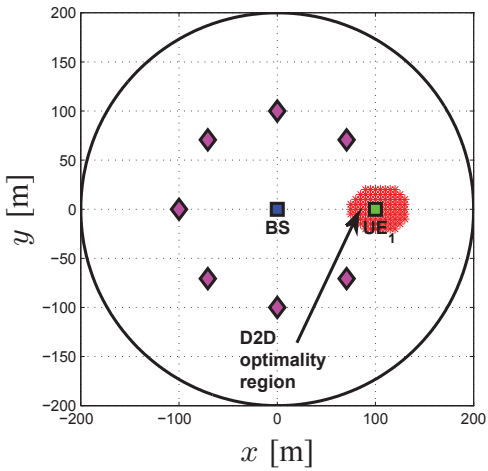

Fig. 7. D2D optimality area in the shared spectrum case with only path-loss information for (P2) with $N=8$ and $M=7$.

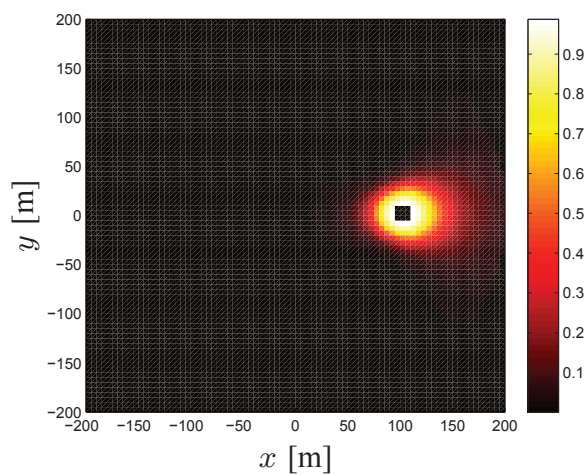

Fig. 9. Probability of D2D mode optimality in the shared spectrum case with fading for (P2) with $N=8$ and $M=7$.

[6] K. Doppler, C. Yu, C. Ribeiro, and P. Jänis, "Mode selection for deviceto-device communication underlaying an LTE-advanced network," in Proc. IEEE Wireless Commun. Network. Conf. (WCNC), Sydney, Australia, Apr. 2010.

[7] Z. Liu, T. Peng, S. Xiang, and W. Wang, "Mode selection for device-todevice (D2D) communication under LTE-advanced networks," in Proc. IEEE Int. Conf. on Commun. (ICC), Ottawa, Canada, Jun. 2012, pp. 5563-5567.

[8] E. Björnson and E. Jorswieck, "Optimal resource allocation in coordinated multi-cell systems," Foundations and Trends in Communications and Information Theory, vol. 9, no. 2-3, pp. 113-381, 2013.

[9] D. Gesbert, M. Kountouris, R. Heath, C. Chae, and T. Salzer, "Shifting the MIMO paradigm," IEEE Signal Process. Mag., vol. 24, no. 5, pp. 36-46, Sep. 2007.

[10] H. Xing and S. Hakola, "The investigation of power control schemes for a device-to-device communication integrated into OFDMA cellular system," in Proc. IEEE Int. Symp. on Personal, Indoor, Mobile Radio Commun. (PIMRC), Istanbul, Turkey, Sep. 2010, pp. 1775-1780. 